\title{
Hive Relocation Does Not Adversely Affect Honey Bee (Hymenoptera: Apidae) Foraging
}

\author{
Fiona C. Riddell Pearce, Margaret J. Couvillon, and Francis L. W. Ratnieks \\ Laboratory of Apiculture \& Social Insects, School of Life Sciences, University of Sussex, Falmer, Brighton BN1 9QG, UK \\ Correspondence should be addressed to Francis L. W. Ratnieks; f.ratnieks@sussex.ac.uk
}

Received 6 June 2013; Revised 29 July 2013; Accepted 29 July 2013

Academic Editor: Felipe Andrés León Contrera

Copyright ( 2013 Fiona C. Riddell Pearce et al. This is an open access article distributed under the Creative Commons Attribution License, which permits unrestricted use, distribution, and reproduction in any medium, provided the original work is properly cited.

Honey bees, Apis mellifera, face major challenges including diseases and reduced food availability due to agricultural intensification. Additionally, migratory beekeeping may subject colonies to a moving stress, both during the move itself and after the move, from the bees having to forage in a novel environment where they have no knowledge of flower locations. This study investigated the latter. We moved three colonies housed in observation hives onto the campus from a site $26 \mathrm{~km}$ away and compared their foraging performance to three similarly sized colonies at the same location that had not been moved. We obtained data on (1) foraging performance by calculating distance by decoding waggle dances, (2) hive foraging rate by counting forager departure rate, (3) forage quality by assessing sugar content of nectar from returning foragers, and (4) forager success by calculating the proportion of bees returning to the nest entrance with nectar in their crop. We repeated this 3 times (August 2010, October 2010, and June 2011) to encompass any seasonal effects. The data show no consistent difference in foraging performance of moved versus resident hives. Overall the results suggest that moving to a new location does not adversely affect the foraging success of honey bees.

\section{Introduction}

Beekeepers routinely move colonies of honey bees Apis mellifera L. to pollinate crops and to take advantage of asynchrony in nectar flows. For example, in the United Kingdom some hives are moved into apple (Malus domestica Borkh.) farms for pollination in early April and onto heather (Calluna vulgaris) moors in late July for obtaining heather honey. In the USA, hives are moved from as far away as Florida to pollinate California almonds in February, a distance of 3000 miles that will take a minimum of 2 to 3 days by truck. Bees are also moved extensively in many other countries (e.g., Turkey [1]). Moving hives has been suggested as an aggravating factor in the decline in colony numbers in the United States [2-4].

Honey bees are an important commercial crop pollinator $[4,5]$. Although the number of managed hives has increased by about $45 \%$ globally since 1961 , the rate of expansion of pollinator-dependant crops is greater than the increase in the number of managed hives, creating increased demand for pollination ([6], although see [7]). In addition, the number of managed hives has declined in Europe and North America, at an average of $1.79 \%$ annually [6]. This potential pollinator shortage is most serious in the USA, where insect pollinated crops are widely grown. For example, the California almond crop, which currently uses over 1 million bee hives for pollination, is worth over $\$ 1.6$ billion annually [4].

Honey bee diseases [8] and the reduction of available bee forage due to agricultural intensification [9-11] are two important challenges facing honey bees. Additionally, beekeeping practices such as hive relocation may also cause undesirable consequences. Close contact of colonies during transport can increase the likelihood of horizontal transfer of pathogens and pests among colonies, and hive movement can spread any newly introduced pathogen in a new geographic area [12]. The process of transport may directly cause stress, leading to brood mortality [13]. Additionally, being moved to a novel environment requires the bees to discover new foraging locations. Previously it was shown that relocation of hives to a new apiary site can cause significant increase in the disorientation and loss of bees via drifting, particularly on the first day after the move [14]. Moving a colony of bees from a familiar landscape into one they have never experienced may hamper the bees' ability to rapidly locate food. 
The aim of this study was to investigate the effect of relocation on colony foraging. To do this, we compared relocated and control colonies for four indicators of foraging success: number of bees leaving the hive, proportion of returning bees with nectar, duration of the straight run of the waggle dance which encodes foraging distance [15], and nectar concentration. The results show no consistent effect of relocating hives on the foraging performance of moved colonies versus resident control colonies.

\section{Materials and Methods}

2.1. Study Colonies and Experimental Setup. Each trial used six A. mellifera colonies, each housed in a glass-walled observation hive with three deep Langstroth frames. Each hive was connected to the outside via a tube through the laboratory wall. Colonies were set up in the laboratory several weeks or more before a trial. Each had a laying queen, two frames of brood each with patches covering approximately half the frame, and sufficient worker bees to cover the frames. Each hive had half a frame of capped honey but also had space for further food storage.

Three "resident" colonies were located in the Laboratory of Apiculture and Social Insects (LASI) at the University of Sussex campus in East Sussex. Three weeks or more before a trial, three "moved" colonies of similar size were established at the Royal Botanic Gardens Wakehurst Place, near Ardingly, West Sussex, and allowed to forage naturally. The Wakehurst Place "moved" colonies were therefore $26 \mathrm{~km}$ from LASI, which makes the foraging range of the two locations nonoverlapping [16]. This is important because the "moved" colonies, once they are relocated to LASI, must have no experience of the area.

Data were gathered from the 6 colonies ( 3 moved and 3 resident) for 2 days (b1 and b2) prior to the relocation of the moved hives to LASI to establish baseline data on foraging performance. Moved hives were then carefully loaded and transported $(<1 \mathrm{~h})$ by car in the evening, to avoid losing foragers, and set up at LASI. Observation hive entrances were $0.9 \mathrm{~m}$ or more apart, each with a distinctly coloured and patterned surround $(50 \times 50 \mathrm{~cm})$ to aid learning of nest location and reduce drifting. Data collection resumed the next day (foraging day 1) on all 6 colonies, now all located at LASI.

\subsection{Choice of Foraging Performance Indicators and Trial} Seasons. We chose four indicators of foraging performance: waggle run duration, which encodes distance [15, 17]; crop nectar concentration in nectar foragers, which is a measure of forage quality [18]; returning bee forage success (whether or not their crops are empty); and departing bees per minute.

For bee departures per minute, one possible outcome could be that colonies with no information on local foraging locations (moved colonies) would send a greater proportion of bees into the field to locate resources. Alternatively it may be that moved hives would show lower departure rates, as they do not know where flowers are. We expect the resident colonies with clear, filtered, public, and private information on available forage should be exploiting the landscape efficiently $[19,20]$.

Waggle run duration encodes foraging distance $[15,17]$ and is useful in measuring efficiency, as flying is costly in terms of energy expenditure and increased predation risk [18]. Honey bees only forage at greater distances when food is in short supply $[11,18]$. We anticipated that moved colonies would spread their foraging efforts over a wider range in an attempt to locate the resources, especially immediately after the move, because they would not yet have the benefit of local knowledge of where to find the best resources. In other words, we expect the move to compromise the optimality of foraging efficiency, which would be reflected in greater communicated distance. In contrast, the resident colonies would already have such information and be foraging over shorter distances. Alternatively, perhaps resident colonies, with knowledge of the most profitable resources, go further, but they bring back better quality forage.

Nectar concentration is a measure of forage quality, as sugar is the main energy source for a honey bee colony, and honey bees are very sensitive to this in their foraging $[15,18,19]$. A crop full of sugar-rich nectar is worth more to the colony than the same volume of low-sugar nectar. Honey bee colonies should therefore aim to collect nectar with high sugar content. We predicted that moved colonies immediately after relocation would initially bring back lower quality nectar than moved colonies until they discover the better quality nectar sources.

For our location and for many other temperate areas, the high summer (early July-late August) flowers are in less abundance compared to spring $[11,16,21]$. Therefore, we predicted that during the August trial, foraging-moved bees would take longer to adjust to the new foraging site than in June, when weather is normally conducive to foraging, and flowers are more abundant. In contrast, during October, flowering ivy is the major source of forage in the study area [22] and is locally abundant, so it should be that moved bees are able to forage comparably to resident colonies quickly, although weather conditions may have more of an impact.

2.3. Collection of Performance Indicator Data. Bees leaving the entrance were counted for 30 minutes per day per hive. 30-minute counting periods were initiated at 0900, 1200, and $1500 \mathrm{~h}, 10$ minutes per hive with 2 observers working simultaneously on different hives.

Nectar quality was determined by using a refractometer (Kruss HR25/800, $21^{\circ} \mathrm{C}$ ) to measure the percentage of sugar in the crop contents of bees returning to each hive. Hive entrances were blocked, and ten returning bees (without visible pollen) from each hive were captured three times daily at 0900,1200 , and $1500 \mathrm{~h}$. Nectar was expelled from the bees' crop by applying gentle pressure to the abdomen of a chilled (immobile) bee. The droplet emerging from the mouth was analysed. Bees were unharmed and were released to resume foraging. Success rate was defined as the percentage of these returning bees with a measurable amount of nectar in their crop.

We decoded waggle dances to determine foraging distances from the waggle run, which is the information-rich 


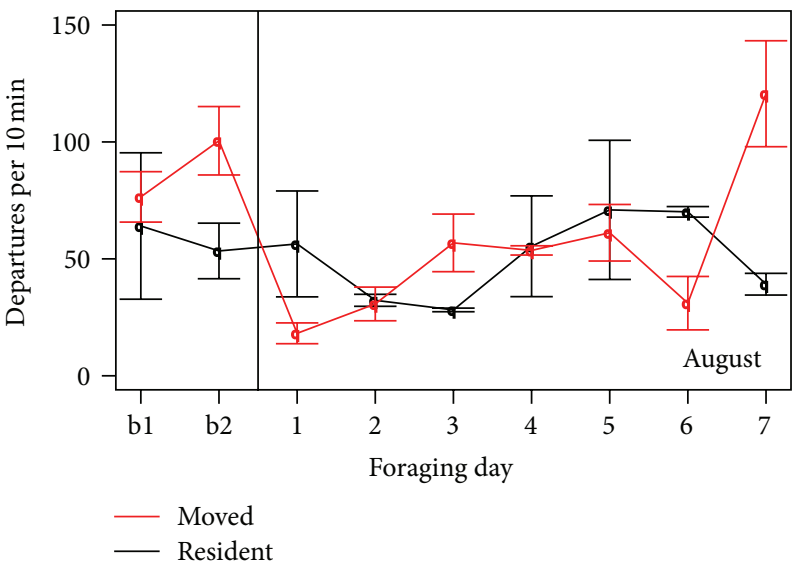

(a)

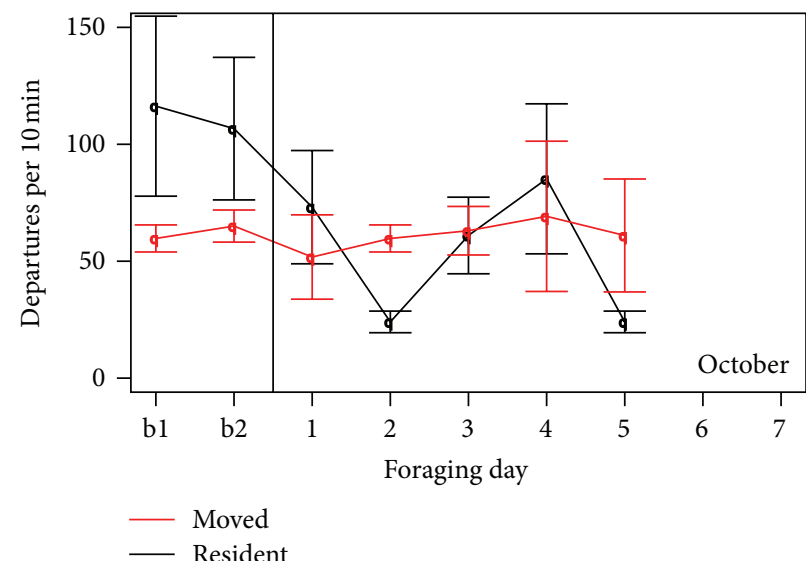

(b)

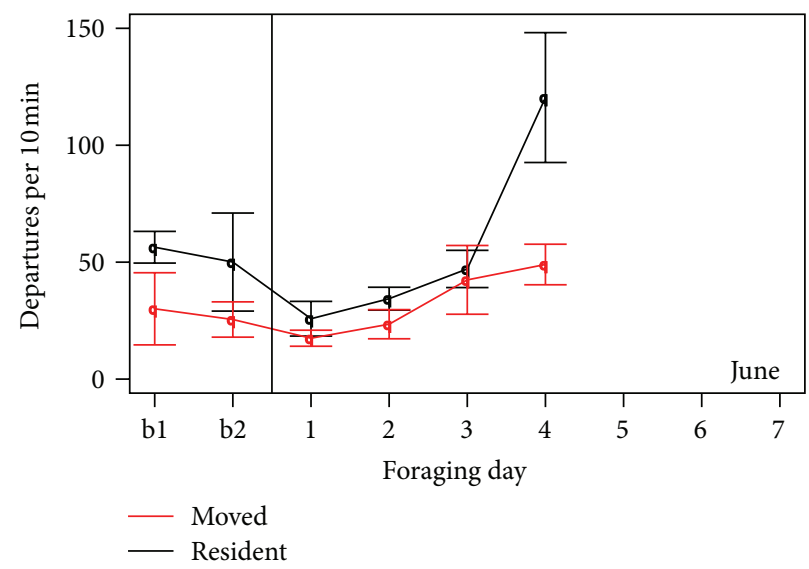

(c)

Figure 1: (a) Mean number of worker bees departing per minute during the three trials of August, October, and June. Days b1 and b2 are the two days immediately before the "moved" hives were relocated to the same location as the resident hives. Error bars are s.e.m. between hives ( 3 resident (black) and 3 moved (red)). Vertical line represents the day the hives were moved.

portion of the dance $[17,23]$. Hives were filmed during three periods each day (0900-1000, 1200-1300, and 1500-1600) using CCTV cameras (Sony Super HAD 27X VHR30) to record the waggle dances made by returning foragers. Footage was then uploaded to an iMac computer. On playback, the duration of the waggle phase was determined to the nearest frame ( $1 / 25$ second) using the timestamp in the video analysis software (MPEG streamclip v1.9.2) [24]. Videos were made August 23,- September 3, 2010, October 7-13, 2010, and June $7-14,2011$. Days where bees did not forage due to bad weather were excluded from the analysis.

We decoded the duration of the waggle run, as this is more accurate than using the whole dance circuit, given that the return phase of the dance circuit can vary in length due to factors such as resource quality $[17,23,24]$. Dances were decoded according to previously published protocols [24], with only the cameras and video playback software differing.

\section{Results}

3.1. Departing Bees per Minute. Figure 1(a) shows the mean number of bees departing the hive entrances for the 6 study colonies in the 3 trial periods before and after moving. For moved colonies, in all three trials the relative departure rates dropped from before (b1 and b2) to first day after relocation (foraging day 1; 80.1, 32.3, and 37.1\% decreases in August, October, and June, resp.). However, the rates for resident colonies also decreased $(3.95,16.96$, and $51.5 \%)$ over the same time period (Figure 1). There was no significant difference between the moved and resident colonies' departing worker rates when we look at differences in bl (day immediately before the move/before vertical line in graph) from day 1 (day immediately after the move/after vertical line in graph; Mann-Whitney, $W=7.0, P=0.19$; Figure 1). In fact, there is no significant difference between the moved and resident colonies' departing worker rates on foraging day 1 (first day after the move) in August (Kruskal-Wallis, $H=3.61, P=$ 0.57 ), October (Kruskal-Wallis, $H=0.44, P=0.507$ ), or June (Kruskal-Wallis, $H=0.38, P=0.535$ ). There was also no significant difference in the number of bees departing per minute on any day after the move other than day 6 of the August trial (Kruskal-Wallis, $H=4.26, P=0.039$ ) and day 4 for the June trial (Kruskal-Wallis, $H=10.39$, $P=0.001)$. 


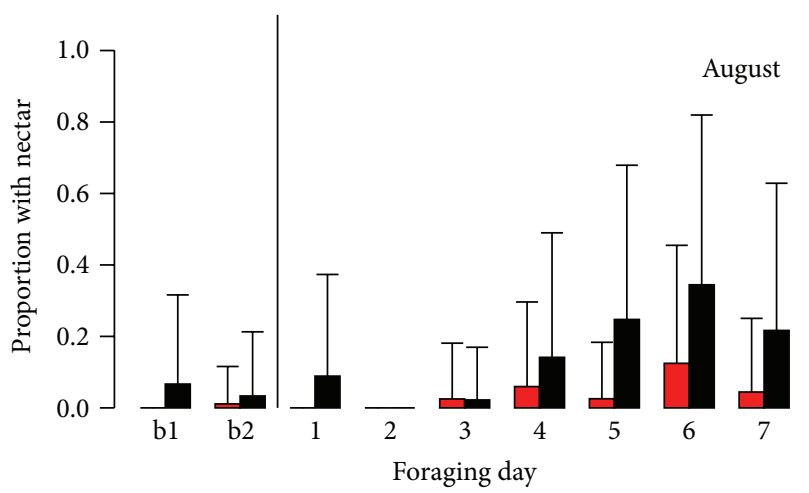

(a)

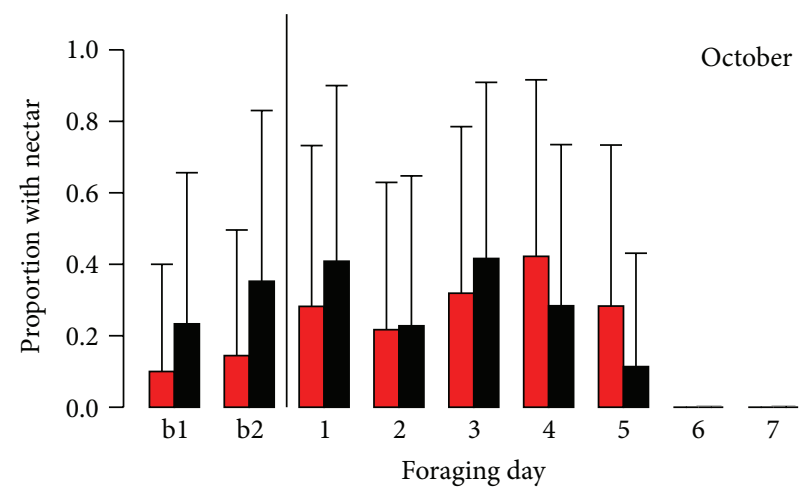

(b)

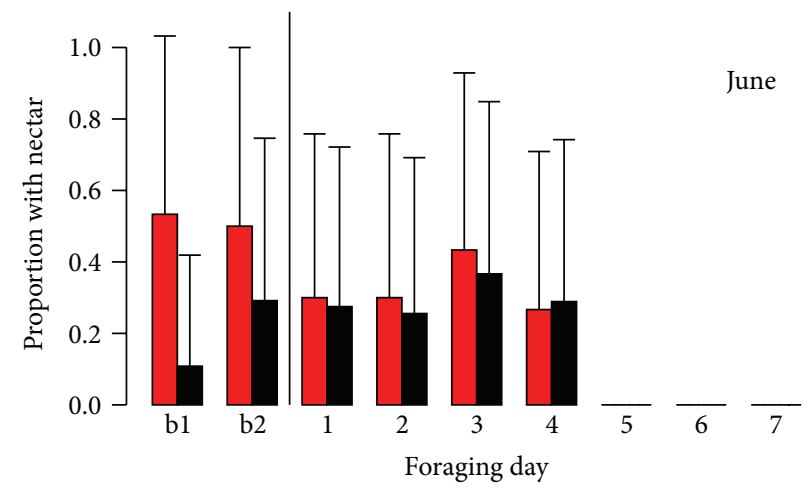

(c)

FIGURE 2: Proportion of returning worker bees with detectable nectar amounts in their crop in the August, October, and June trials before (b1 and b2) and after (days 1-7) the move for both resident (black) and moved (red) hives. Vertical line represents the day the hives were moved.

\subsection{Proportion of Returning Workers with Nectar Loads.} Overall, low proportions $(<0.5)$ of returning workers carried nectar, and this metric showed large fluctuations (Figure 2). Overall success decreased after the move for both resident and moved colonies in August (mean of days b1 and b2 = $5 \%$, mean of days 1 and $2=4.45 \%$ for resident; mean of b1 and b2 $=0.55 \%$, mean of days 1 and $2=0 \%$ for moved colonies), increased for both resident and moved in October trial (resident, $29.3 \%$ to $31.9 \%$; moved, $12.2 \%$ to $39.1 \%$ ), and increased for resident colonies and decreased for moved colonies in June trials (resident, $10.6 \%$ to $31.1 \%$; moved, $35.6 \%$ to $22.2 \%)$.

Specifically, we compared moved and resident hives immediately before the move (foraging day b2) and immediately after the move (foraging day 1). Despite these fluctuations in foraging success, the resident colonies were not consistently more successful than moved colonies (Figure 2). In August on foraging day 1, moved colonies did possess significantly more successfully returning nectar foragers (twoway contingency, $\chi^{2}=12.8, \mathrm{df}=1$, and $P=0.003$ ); however, in October and June, this relationship was highly nonsignificant (October: $\chi^{2}=0.06, \mathrm{df}=1$, and $P=0.8$; June: $\chi^{2}=0.317$, $\mathrm{df}=1$, and $P=0.57$ ), with in June, the trend being for a higher proportion of moved returning foragers to have nectar compared to resident. When we looked at specifically at foraging day b2 versus 1, in August, both the resident and moved colonies performed significantly worse on the day after the move (resident: $\chi^{2}=12$, df $=1$, and $P=0.0005$; moved: $\chi^{2}=6.61, \mathrm{df}=1$, and $\left.P=0.01\right)$. In October and June, the resident colonies performed better on the day after the move, but not significantly so (October: $\chi^{2}=0.87, \mathrm{df}=1$, $P=0.35$; June: $\left.\chi^{2}=0.11, \mathrm{df}=1, P=0.74\right)$. The moved hives performed significantly better on the day after the move in October (October: $\chi^{2}=745, \mathrm{df}=1$, and $P=0.006$ ), and in June, the moved hive performance on the day after the move was slightly worse, but not significantly so (June: $\chi^{2}=0.54$, df $=1$, and $P=0.46$ ).

3.3. Waggle Run Duration. On the first foraging day after the move, moved and resident hives foraged at similar distances, as indicated by similar waggle run durations, in the August and June trials (one-way ANOVA: August, $F=1.54, P=$ 0.22 ; June $F=0.24, P=0.631$; Figure 3 ). However, in the October trial, the resident hives performed no dances on day 1, while the moved hives did dance. In August, the resident and moved colonies foraged over similar distances on all days other than days 5 and 6 , when the moved colonies foraged at greater distances than the resident colonies (Oneway ANOVA: $F=4.55, P=0.037 ; F=12.38, P=0.001$ for days 5 and 6, resp.) and over a greater range of distances (mean waggle phase duration, resident $=1.76 \mathrm{~s}$, moved $=$ $2.27 \mathrm{~s}$; range, resident $=3.75 \mathrm{~s}$, moved $=7.86 \mathrm{~s}$ ).

During October, the foraging distances of resident and moved colonies were different before the move but not 


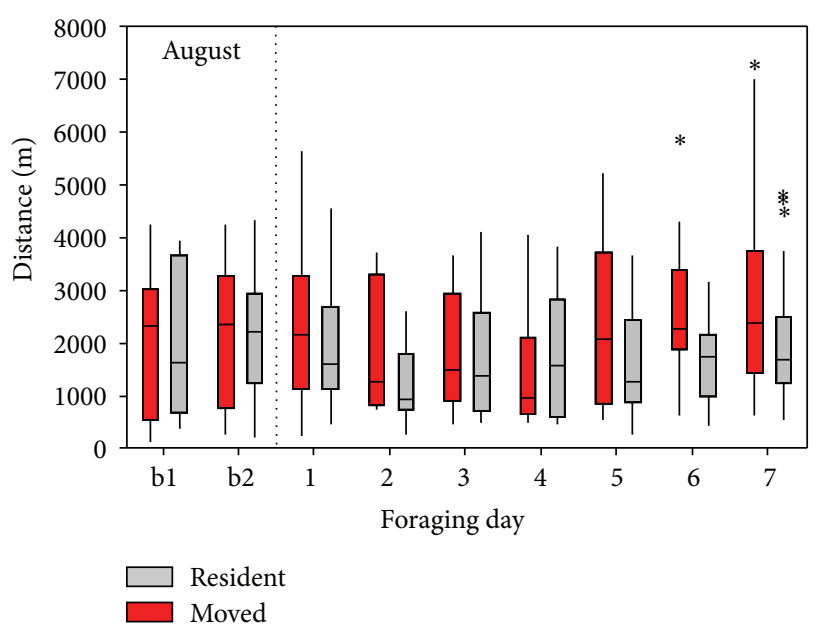

(a)

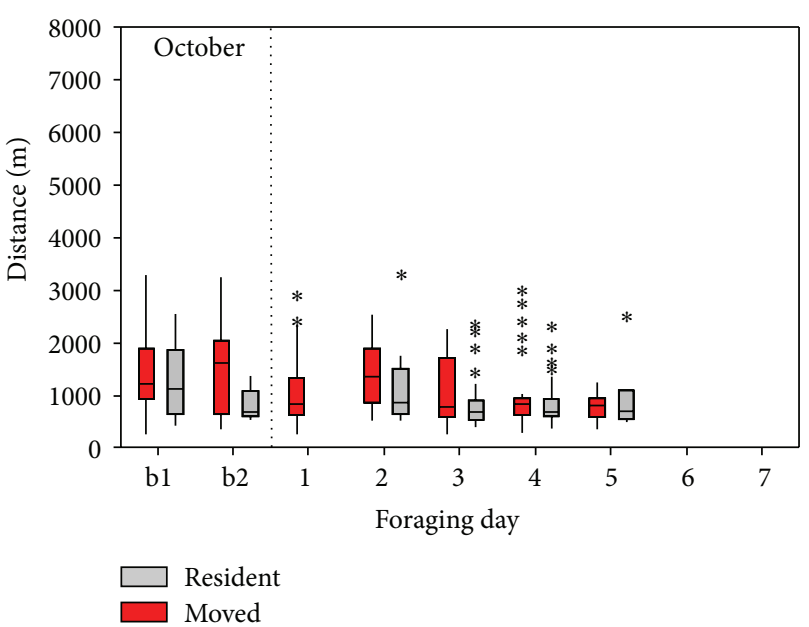

(b)

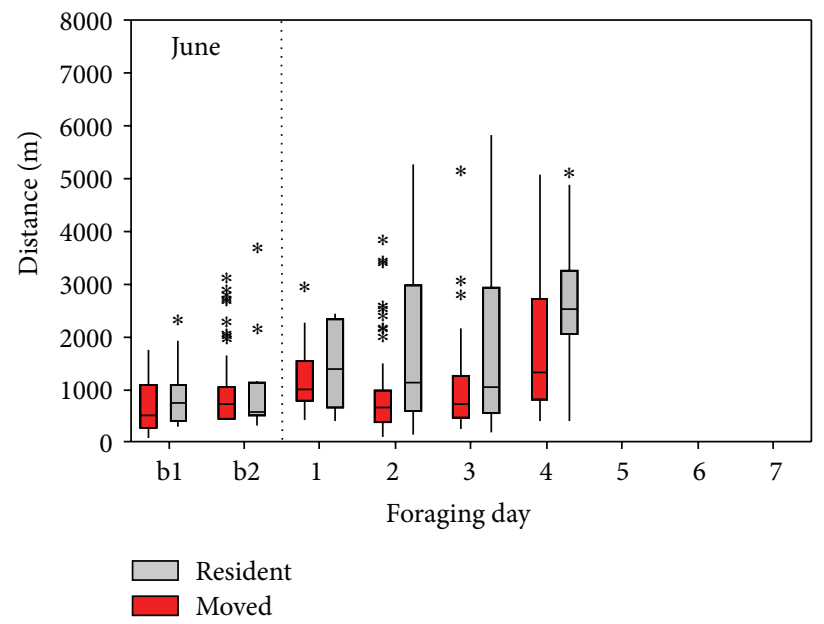

(c)

FIGURE 3: Durations of waggle runs of dancing bees in August, October, and June trials. Longer waggle runs indicate greater foraging distance. Days b1 and b2 are the two days immediately before the "moved" hives were relocated, in the evening after foraging had ended, to the same location as the resident hives. Dashed grey line indicates relocation, and stars represent a single datum point that does not fall within the whisker range.

significantly different on any day after the move $(F=0.21$, $P=0.646$ ) (other than foraging day 1 , see above). In June, resident colonies foraged at greater distances on days 2,3 , and 4 (mean, resident, $1.9 \mathrm{~s}$; moved, $1.3 \mathrm{~s}$ ) than moved hives (oneway ANOVA: $F=18.56, P<0.005 ; F=6.73, P=0.011$; $F=8.12, P=0.005$ on days 2,3 , and 4 , resp.).

3.4. Nectar Concentration. Moved hives failed to return with measurable nectar until day 3 after the relocation in August. The resident hives collected nectar with a mean concentration of $18.2 \%$ and $18.6 \%$ on days b1 and 1 but failed to collect nectar on days b2 and 2 (Figure 4). There were fluctuations in the concentration of nectar collected between hives and trial periods, but moved and resident hives brought back similar concentrations of nectar after the move with the exception of day 4 in August where moved hives found better quality nectar $(56.8 \%$ versus $45.1 \%$; one-way ANOVA: $F=15.29$, $P=0.001)$ and day 3 in October where the resident hives brought back higher quality nectar (32.2 versus $28.0 \%$; oneway ANOVA: $F=4.45, P=0.037$ ). In October, moved hives found poorer quality nectar before the move than resident colonies (one-way ANOVA: b1, $F=31.18, P<0.000$; b2, $F=21.66, P<0.001)$; however, on the first day after the move, there was no significant difference between the nectar concentration found by moved and resident colonies (oneway ANOVA: $F=0.13, P=0.721$ ).

\section{Discussion}

Our results show no consistent differences in the four measures of foraging performance for resident colonies versus colonies relocated into the same location. There were differences in foraging performance before the move, such as lower mean nectar concentration for moved hives in October (14.5\% versus $35 \%$ ) and lower mean departures per minute for the moved hives than the resident hives in June (12.4 


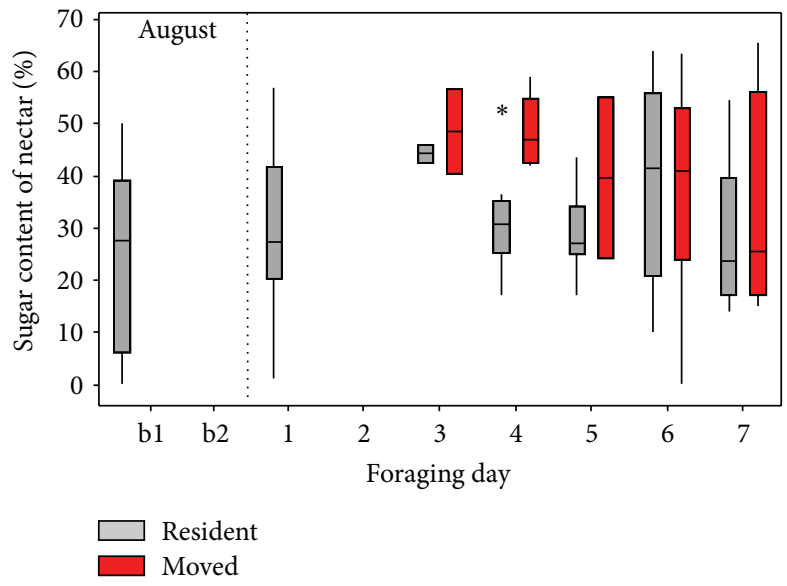

(a)

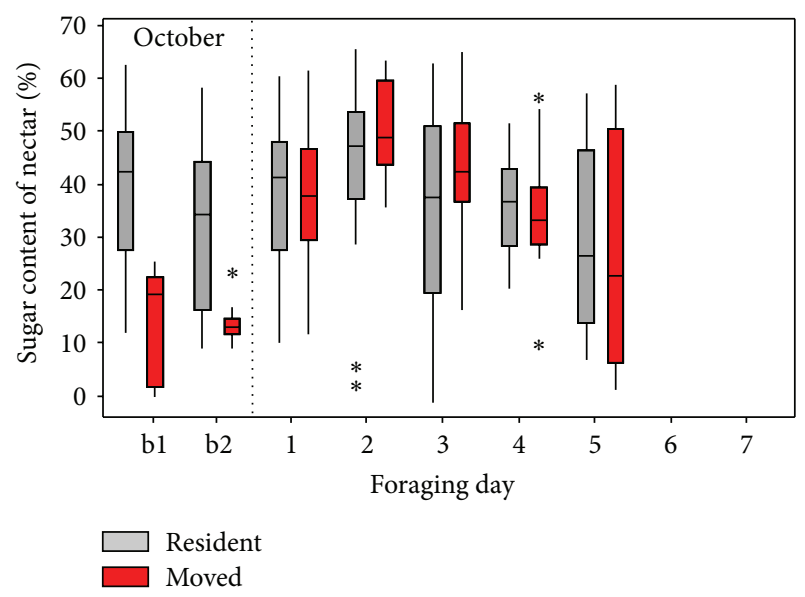

(b)

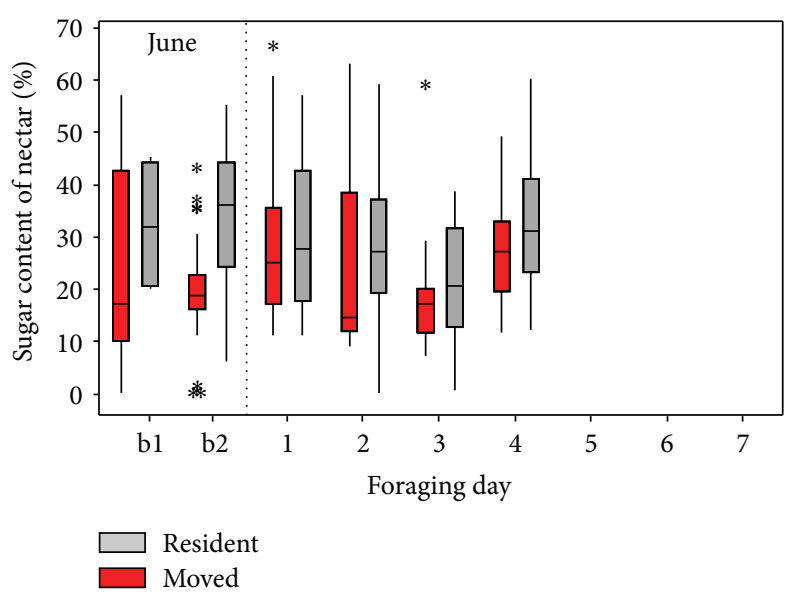

(c)

FIGURE 4: Sugar concentration of nectar in returning worker bees in the three study periods, August, October, and June. Days b1 and b2 are the two days immediately before the "moved" hives were moved, in the evening after foraging had ended, to the same location as the resident hives. Dashed grey line indicates relocation, and stars represent a single datum point that does not fall within the whisker range.

versus 6.5), presumably due to the difference in location and food availability. Overall, climatic conditions and seasonal resource availability were more likely an influence on the foraging performance of both moved and resident colonies than the relocation to a novel environment. Bees were rapidly able to find new and high quality sources of food after the move, with moved hives bringing back similar quality of nectar compared to resident colonies on the first day after the move in October and June (for August, see below). If foraging performance was poor, it was poor for both resident and moved colonies.

In the case of departure rates, there was no consistent trend for the moved hives to have lower rates with the exception of day 1 in the August trial (Figure 1(a)). Although departure rates of moved colonies dropped immediately after the move, they also dropped for the resident colonies, suggesting that weather conditions were more likely to be the cause with $0.2 \mathrm{~mm}$ and $0.6 \mathrm{~mm}$ of rain on b1 and b2 in August and $0 \mathrm{~mm}$ on days 1-7. This would be because, after rainfall, nectaries are often washed out, and pollen is too soggy to collect. We found a great deal of overlap in the changes in departure rates of the colonies in all three trials, indicating that moved and resident colonies were changing their departure rates by similar proportions (Figure 1(b)).

There was no clear or consistent trend in the success of returning workers other than the fact that the success was surprisingly low, with less than $50 \%$ of returning workers having nectar in their crop. In August workers experienced the lowest success, and less than $10 \%$ of both resident and moved returning workers had measurable nectar, with both groups having $0 \%$ success on day 3 . Cool, damp weather reduces nectar availability to insects (Peat and Goulson, 2005) and was almost certainly an important factor in poor foraging performance in the August trial in addition to August being the most challenging month of the year for bees to find food, with the bees needing to travel at greater distances in our region [11], which is temperate.

In the October and June trials, during which the weather was drier than in the August trial $(1 \mathrm{~mm}$ of rain fell on day 1 and $0.2 \mathrm{~mm}$ on day 2 in June, but this was overnight and did not interrupt foraging), success was greater, but never more than $50 \%$. The nectar collected on successful 
foraging days after the moves was of similar concentrations for resident and moved hives on all days other than day 6 in August and day 4 in June. The nectar collected by the moved hives before the move was of lower concentration than that collected after the move by both groups and before the move by resident colonies. However, on the first day after the move, both groups had located similar quality nectar. Variation in plant availability at the two sites can account for this difference. Greater coverage of ivy (Hedera helix), which is a major food source for honeybees from early September to early November [22], was observed at the laboratory than at Wakehurst Place.

It is interesting to examine departure rate data in light of the proportion returning with nectar. If departing bees also included large numbers of scouts, we would expect the moved hives to experience higher departure rates than before the move compared to the resident hives; additionally, we would expect noticeably fewer returning bees to contain nectar in crops. However, the success of returning foragers did not follow a clear trend, with resident colonies always returning a higher proportion of bees with full crops; additionally, there were no clear increases in the number of departing foragers from moved hives.

In the case of foraging distance, as shown by dance decoding, foragers from both moved, and resident colonies foraged at similar distances during most foraging days. On the days where the resident and moved hives did forage at different distances, the moved hives foraged at greater distances than resident hives in August (days 5 and 6), but the reverse was the case in June (days 2, 3, and 4). In October, the resident hives performed no dances on day 1 , while the moved hives danced normally. One possible explanation is that the resident hives did not dance, as they knew where flowers were, but they were not sufficiently exciting to elicit dancing, whereas the threshold for the moved hives was lower, so they did dance.

Sherman and Visscher found that the waggle dance was more important to colony fitness in southern California under winter foraging conditions, with colonies prevented from performing oriented waggle dances losing more weight [25]. We found that colonies performed more dances per day in June, and there was no significant difference in the mean number of dances per day for either resident or moved hives before or after the move in August or October. A possible explanation for this is that there was more dancing in June because there was more available food, whereas although dancing may be more important in August, there were fewer sources available worth advertising.

Why might it be that we did not see a significant effect of relocation on the foraging performance of the moved bees versus the resident bees? A colony of bees has many foragers in the field at once, up to $25 \%$ of the colony's workers [19]. These foragers collect information on food availability over an area surrounding the hive of up to $100 \mathrm{~km}^{2}$ [16] and share this information with their nestmates via the waggle dance. Seeley [26] showed that if a food patch $\left(100 \mathrm{~m}^{2}\right)$ is within $1000 \mathrm{~m}$ of the hive, there is a $70 \%$ chance of the colony locating it. This chance drops to $50 \%$ for a patch located $2000 \mathrm{~m}$ from the hive entrance. Once a resource has been located by a scout, the number of visiting foragers increases rapidly as recruits are informed via the waggle dance [15]. Seeley and Visscher also showed that the waggle dance allows colonies to locate better quality food and that they can do so quickly. Scout bees are able to discover a flower patch $610 \mathrm{~m}$ away within 200 minutes of the resources being placed. this 200 minutes is lessened for closer resources [27]. Large numbers of recruit bees, presumably directed by the scout's waggle dances, then arrived within 50 minutes of its discovery by the scout. This shows that honey bee colonies have considerable ability rapidly to track both spatial and temporal changes in food availability. If food is available in the landscape, it is likely that a honey bee colony will locate it. The location of floral resources varies with season, but also from day to day, and even at different times of day, as some plants only produce nectar at certain times of day [28]. The fact that honey bee colonies have evolved mechanisms to track these changes may mean that a colony moved to a novel environment is not at a great disadvantage. In addition, honey bee colonies naturally change their location when a swarm establishes a new nest. In European A. mellifera, the new nest is within a few kilometres of the natal nest [29] However, swarms of African A. mellifera [30] and Asian Apis dorsata [31] migrate longer distances.

This study involved moving bees to a novel location, which was similar in terms of climate and available forage. It is possible that moving bees over much larger distances into different climatic conditions and resource availability may have a much greater impact on foraging efficiency. It is also possible that it may take longer to adjust to a new foraging location if the plant species are different from the known location, as odour memory plays an important role in foraging and location of food sources [23, 32]. It was shown that after a move, foragers tended to forage on species they had previously visited if they were available [33].

Our study has produced some encouraging results. With many studies focusing on factors that can potentially harm bees, such as pesticides, pest and pathogens, and lack of forage $[8,11,34]$ and much of the media focused on the decline in honey bees and other pollinators, it is reassuring to find a factor which seems not be detrimental to honey bee colonies.

\section{Acknowledgments}

Fiona C. Riddell Pearce was funded by Waitrose, and Margaret J. Couvillon was funded by the Nineveh Charitable Trust. Thanks are due to Ashleigh Bates and Aurelie Croizille for their help in decoding dances.

\section{References}

[1] I. Kandemir, M. Kence, and A. Kence, "Genetic and morphometric variation in honeybee (Apis mellifera L.) populations of Turkey," Apidologie, vol. 31, no. 3, pp. 343-356, 2000.

[2] B. P. Oldroyd, "What's killing American honey bees?" PLoS Biology, vol. 5, no. 6, article e168, 2007.

[3] F. L. W. Ratnieks and N. L. Carreck, "Clarity on honey bee collapse?” Science, vol. 327, no. 5962, pp. 152-153, 2010. 
[4] M. Berenbaum, Committee on the Status of Pollinators in North America, and National Research Council, Status of Pollinators in North America, The National Academies Press, Washington, DC, USA, 2007.

[5] K. S. Delaplane and D. F. Mayer, Crop Pollination by Bees, CABI, New York, NY, USA, 2000.

[6] M. A. Aizen and L. D. Harder, "The global stock of domesticated honey bees is growing slower than agricultural demand for pollination," Current Biology, vol. 19, no. 11, pp. 915-918, 2009.

[7] L. G. Carvalheiro, W. E. Kunin, P. Keil et al., "Species richness declines and biotic homogenisation have slowed down for NWEuropean pollinators and plants," Ecology Letters, vol. 16, no. 7, pp. 870-878, 2013.

[8] D. L. Cox-Foster, S. Conlan, E. C. Holmes et al., "A metagenomic survey of microbes in honey bee colony collapse disorder," Science, vol. 318, no. 5848, pp. 283-287, 2007.

[9] S. Kluser and United Nations Environment Programme, Global Honey Bee Colony Disorders and Other Threats to Insect Pollinators, UNEP Emerging Issues, United Nations Environment Programme, Geneva, Switzerland, 2011.

[10] P. A. Matson, W. J. Parton, A. G. Power, and M. J. Swift, "Agricultural intensification and ecosystem properties," Science, vol. 277, no. 5325, pp. 504-509, 1997.

[11] M. J. Couvillon, R. Schürch, and F. L. W. Ratnieks, "Dancing bees provide an integrative picture of seasonal foraging challenges," submitted.

[12] E. Crane, Bees and Beekeeping: Science, Practice and World Resources, Heinemann Newnes, 1990.

[13] K. Hackett and United States Department of Agriculture, Colony Collapse Disorder Progress Report, United States Department of Agiculture, Beltsville, Md, USA, 2010.

[14] D. Nelson and S. Jay, "The effect of colony relocation on loss and disorientation of honeybees," Apidologie, vol. 20, no. 3, pp. 245-250, 1989.

[15] K. von Frisch, The Dance Language and Orientation of Bees, Harvard University Press, Cambridge, Mass, USA, 1967.

[16] M. Beekman and F. L. W. Ratnieks, "Long-range foraging by the honey-bee, Apis mellifera L," Functional Ecology, vol. 14, no. 4, pp. 490-496, 2000.

[17] T. D. Seeley, A. S. Mikheyev, and G. J. Pagano, "Dancing bees tune both duration and rate of waggle-run production in relation to nectar-source profitability," Journal of Comparative Physiology A, vol. 186, no. 9, pp. 813-819, 2000.

[18] T. D. Seeley, "Honey bee foragers as sensory units of their colonies," Behavioral Ecology and Sociobiology, vol. 34, no. 1, pp. 51-62, 1994.

[19] T. D. Seeley, The Wisdom of the Hive, Harvard University Press, Cambridge, Mass, USA, 1995.

[20] C. Grüter, E. Leadbeater, and F. L. W. Ratnieks, "Social learning: the importance of copying others," Current Biology, vol. 20, no. 16, pp. R683-R685, 2010.

[21] B. Heinrich and P. H. Raven, "Energetics and pollination ecology," Science, pp. 597-602, 1972.

[22] M. Garbuzov and F. L. W. Ratnieks, "Ivy: an underappreciated key resource to flower visiting insects in autumn," Insect Conservation and Diversity, 2013.

[23] M. J. Couvillon, “The dance legacy of Karl von Frisch," Insectes Sociaux, vol. 59, no. 3, pp. 297-306, 2012.

[24] M. J. Couvillon, F. C. Riddell Pearce, E. L. Harris-Jones et al., "Intra-dance variation among waggle runs and the design of efficient protocols for honey bee dance decoding," Biology Open, vol. 1, no. 5, pp. 467-472, 2012.

[25] G. Sherman and P. K. Visscher, "Honeybee colonies achieve fitness through dancing," Nature, vol. 419, no. 6910, pp. 920-922, 2002.

[26] T. D. Seeley, "The effectiveness of information collection about food sources by honey bee colonies," Animal Behaviour, vol. 35, no. 5, pp. 1572-1575, 1987.

[27] T. D. Seeley and P. K. Visscher, "Assessing the benefits of cooperation in honeybee foraging: search costs, forage quality, and competitive ability," Behavioral Ecology and Sociobiology, vol. 22, no. 4, pp. 229-237, 1988.

[28] R. E. Irwin, L. S. Adler, and A. K. Brody, "The dual role of floral traits: pollinator attraction and plant defense," Ecology, vol. 85, no. 6, pp. 1503-1511, 2004.

[29] T. D. Seeley, Honeybee Democracy, Princeton University Press, Princeton, NJ, USA, 2010.

[30] F. L. W. Ratnieks, "Africanized bees: natural selection for colonizing ability," in The "African" Honey Bee, pp. 213-234, Westview Press, Boulder, Colo, USA, 1991.

[31] J. Paar, B. P. Oldroyd, and G. Kastberger, "Giant honeybees return to their nest sites," Nature, vol. 406, no. 6795, p. 475, 2000.

[32] M. Hammer and R. Menzel, "Learning and memory in the honeybee," Journal of Neuroscience, vol. 15, no. 3 I, pp. 1617-1630, 1995.

[33] J. B. Free, "The effect of moving colonies of honeybees to new sites on their subsequent foraging behaviour," Journal of Agricultural Science, vol. 53, no. 1, pp. 1-9, 1959.

[34] M. Henry, M. Béguin, F. Requier et al., "A common pesticide decreases foraging success and survival in honey bees," Science, vol. 336, no. 6079, pp. 348-350, 2012. 

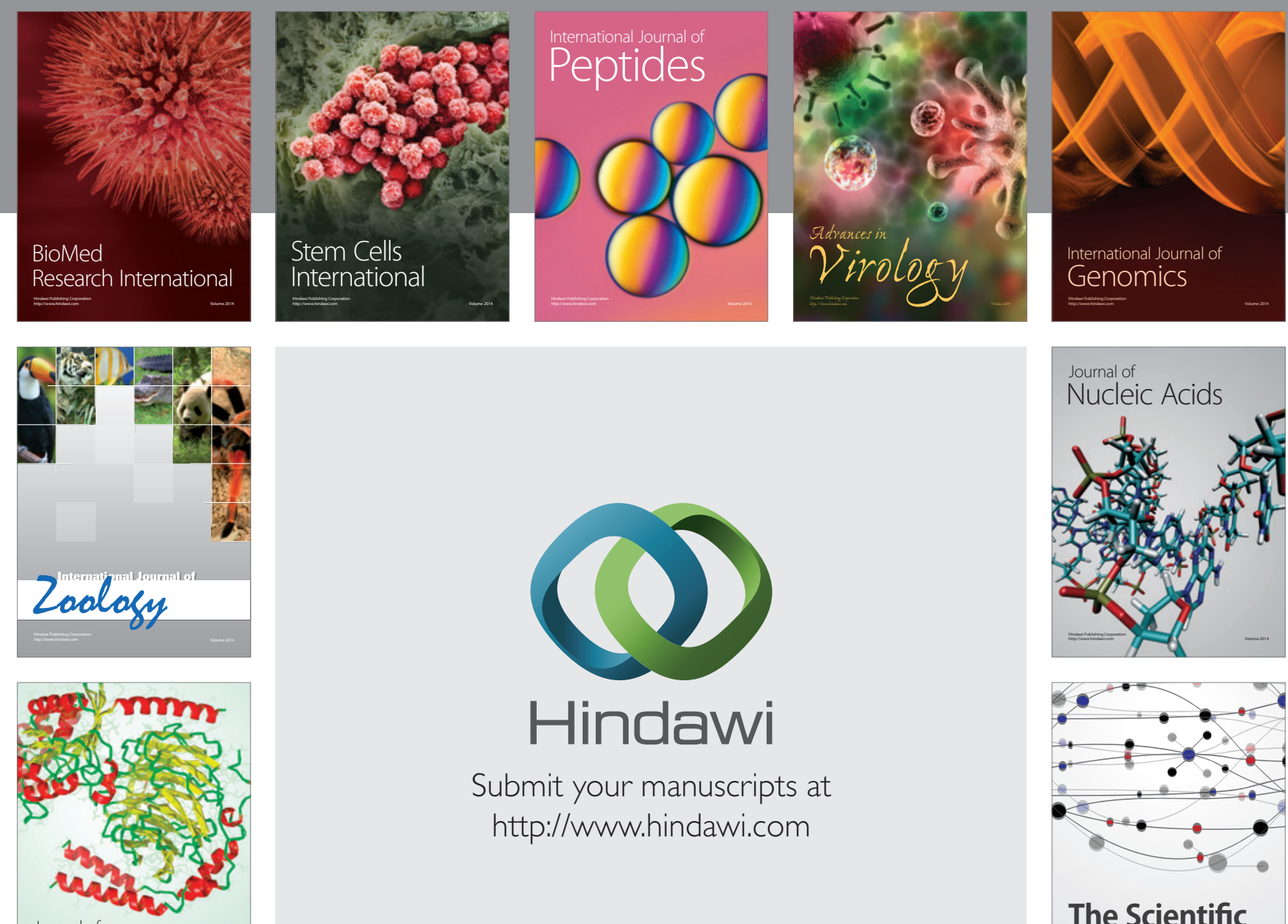

Submit your manuscripts at

http://www.hindawi.com

Journal of
Signal Transduction
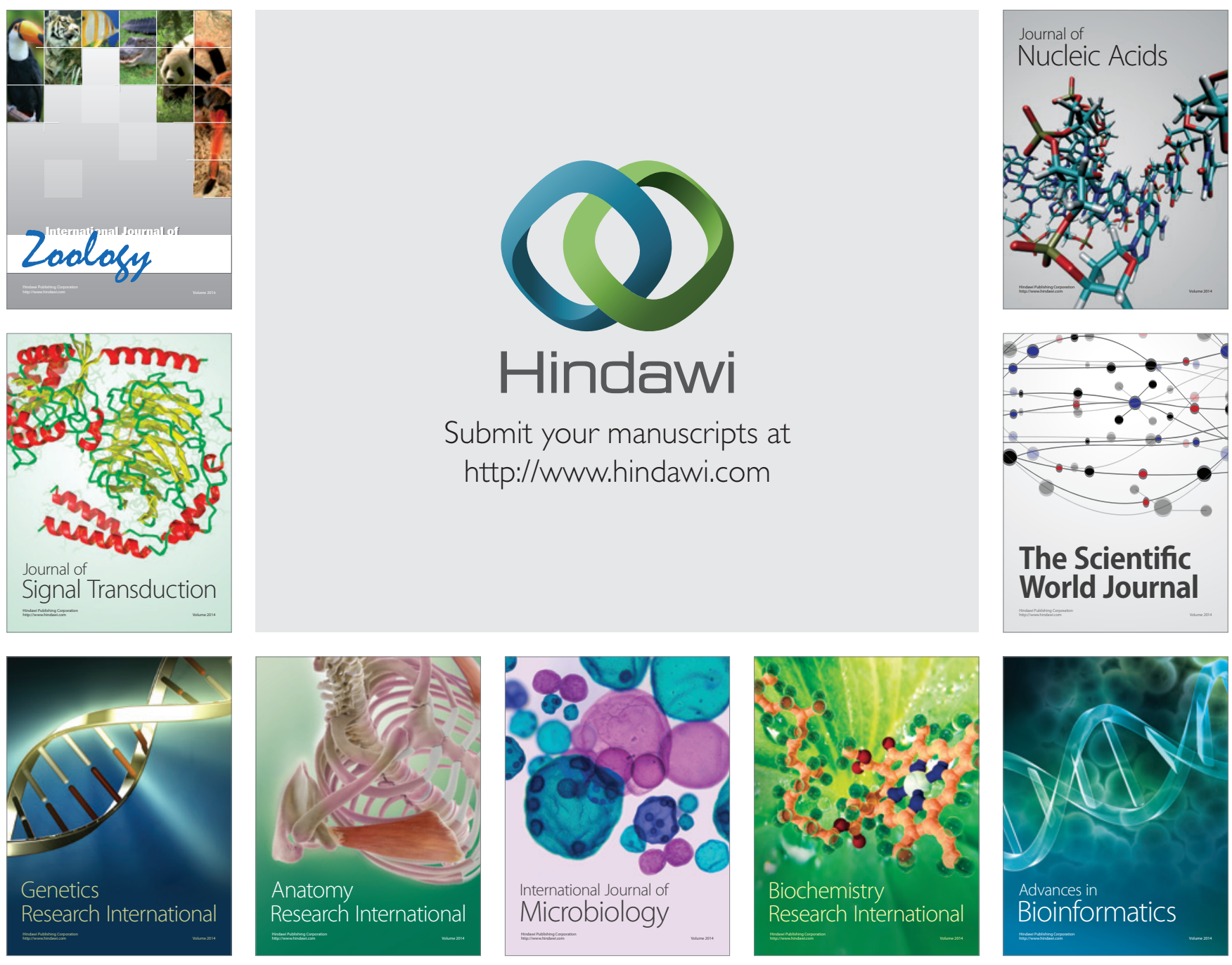

The Scientific World Journal
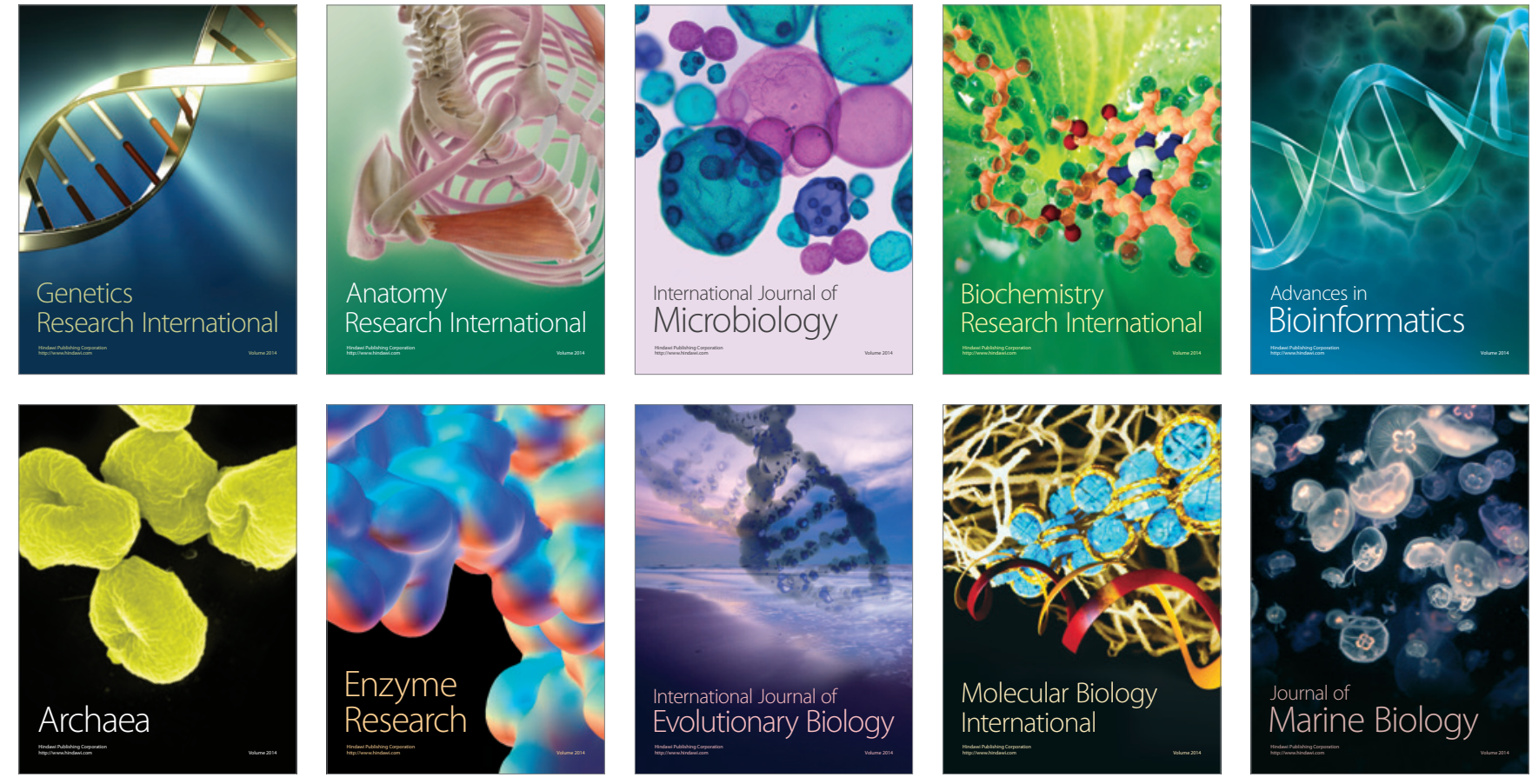